\title{
AVALIAÇÃO TÉCNICA E ECONÔMICA DO FORWARDER NA EXTRAÇÃO DE MADEIRA EM POVOAMENTO DE EUCALIPTO DE PRIMEIRO CORTE
}

\author{
Danilo Simões ${ }^{1}$, Paulo Torres Fenner ${ }^{2}$ \\ ${ }^{1}$ Adm. de Empresas, M.Sc., Depto. de Ciências Florestais, UNESP, Botucatu, SP, Brasil - simoesdanilo@yahoo.com.br \\ ${ }^{2}$ Eng. Florestal, Dr., UNESP, Depto. de Ciências Florestais, UNESP, Botucatu, SP, Brasil - fenner@ fca.unesp.br
}

Recebido para publicação: 20/01/2009 - Aceito para publicação: 09/03/2010

\begin{abstract}
Resumo
O objetivo deste trabalho foi avaliar técnica e economicamente o Forwarder em operação de extração de madeira curta em povoamento de primeiro corte de Eucalyptus grandis. A análise técnica englobou um estudo de tempos e movimentos pelo método contínuo e a produtividade foi determinada através do volume em metros cúbicos de madeira extraída. A análise econômica englobou os parâmetros do custo operacional e custo de extração da madeira. Os resultados referem-se ao rendimento operacional efetivo. As atividades parciais carregamento e descarregamento, juntas, corresponderam a mais de $3 / 4$ do tempo total do ciclo operacional efetivo do Forwarder. O custo operacional foi seguramente influenciado pelos custos de reparo e manutenção. O custo estimado de extração foi de US\$ 0.97 por metro cúbico.

Palavras-chave: Custos de produção; auto-carregáveis; rendimentos operacionais.
\end{abstract}

\begin{abstract}
Operational and economical evaluation of the Forwarder in wood extraction from first cutting eucalyptus. This study aimed to technically and economically evaluate the Forwarder performance in the first cutting wood extraction from a Eucalyptus grandis plantation. Technical analysis included a movement and timing study by continuous method, and the productivity was determined using the extracted wood volume in cubic meters. Economical analysis included operational costs parameters and wood extraction cost. Results referred to the effective operational performance. Loading and unloading activities responded for more than $3 / 4$ of the total time of effective Forwarder operational cycle. Operational costs were influenced by maintenance and repair costs. The estimated cost of extraction was US\$ 0.97 per cubic meter.

Keywords: Production costs; auto loading; operational performance.
\end{abstract}

\section{INTRODUÇÃO}

No Brasil, entre as principais atividades do agronegócio, está a atividade florestal, que vem se consolidando competitivamente, fortalecendo a investigação incessante de conhecimento e de novas tecnologias, a fim de promover condições inovadoras aos diferentes elos da cadeia produtiva do agronegócio florestal, de forma a propiciar inovação, qualidade, desenvolvimento e otimização dos recursos empregados (SIMÕES, 2008).

A fase final da colheita florestal é o transporte primário da madeira, o qual ocorre após a derrubada ou processamento e consiste na movimentação da madeira desde o interior dos talhões até as margens das estradas florestais ou pátios. Nos sistema de toras curtas, geralmente são utilizados os tratores agrícolas autocarregáveis e os forwarders.

A extração da madeira é um dos pontos críticos da colheita, uma vez que o custo de unidade de madeira de uma etapa chega a ser 25 vezes maior que o transporte principal em alguns países. Porém a mecanização exige o uso de equipamentos dimensionados para executarem suas tarefas e que apresentem custos compatíveis, baixo impacto ambiental e proporcionem boas condições de trabalho ao operador (MINETTE, 1988). 
A produtividade das operações de colheita é uma das principais variáveis que condiciona a viabilidade da retirada de madeira dos projetos florestais, sendo, normalmente, inversamente proporcional ao custo por $\mathrm{m}^{3}$ (MALINOVSKI et al., 2006).

Seixas (1986), em artigo sobre planejamento e estudo de sistemas de exploração, ressalta a necessidade de se conhecerem profundamente as atividades desenvolvidas em uma operação florestal, sendo que o levantamento de informações deve servir de base para qualquer interferência que se queira fazer.

Simões; Fenner (2010) afirmam que a realização de estudos sobre as variáveis que influenciam a produtividade das operações florestais tornaram-se imperativas, visando à minimização dos custos e à otimização operacional. A identificação dessas variáveis pode ser realizada por estudos específicos que possibilitem estimar a produtividade, a qual resultará em subsídios para avaliação mais precisa do processo de produção. Portanto, para se intervir no sistema e adotar estratégias de racionalização, é necessário que se conheça, detalhadamente, todo o processo, pois só assim será possível atuar nos pontos deficientes (MINETTE et al., 2008).

Nesse contexto, o estudo teve como objetivo determinar as atividades parciais, estimar o rendimento operacional e custos da operação do forwarder na extração de toras curtas de eucalipto em floresta de primeiro corte.

\section{MATERIAL E MÉTODOS}

\section{Área experimental}

O estudo foi desenvolvido em um povoamento de Eucalyptus grandis de origem seminal, de primeiro corte, com seis anos de idade, num talhão com o mesmo sítio silvicultural (site índex), formado por um povoamento homogêneo e equiâneo que passou pelos mesmos tratos silviculturais.

A área experimental está localizada nas coordenadas geográficas $23^{\circ} 11^{\prime}$ de latitude sul e $48^{\circ} 30^{\prime}$ de longitude oeste, no estado de São Paulo.

O tipo de solo predominante na região é o arenoso de cor castanho-avermelhado, com relevo classificado como plano e com uma altitude média de 650 metros acima do nível do mar.

A área do povoamento estudado, segundo a classificação de Wilhelm Köppen, está localizada em área de clima Cwa, clima temperado quente (mesotérmico), com chuvas no verão e seca no inverno. $\mathrm{O}$ total de precipitação pluvial anual apresenta a média de $1.524,5 \mathrm{~mm}$. A temperatura média anual é de $20,5^{\circ} \mathrm{C}$, sendo $17,2^{\circ} \mathrm{C}$ no mês mais frio e $23,4^{\circ} \mathrm{C}$ no mês mais quente (CUNHA et al., 1999).

A coleta dos dados foi efetuada nos mesmos horários e condições climáticas, as quais se mantiveram sem alterações, com o céu encoberto e com períodos de sol durante o período do estudo.

\section{Descrição do forwarder}

O forwarder empregado no estudo era da marca Valmet, modelo $890.2 / 6 \mathrm{WD}$, com peso aproximado de $16.800 \mathrm{~kg}$, capacidade de carga máxima de $18.000 \mathrm{~kg}$, largura padrão frontal/traseira $2.995 \mathrm{~mm}$, motor modelo Sisu, diesel 74 ETA 6 cilindros, turbo alimentado com intercooler, potência $170 \mathrm{~kW}$ DIN (230 hp) a $1.700 \mathrm{rpm}$, torque $1000 \mathrm{Nm}(104 \mathrm{kpm})$ a 1.200-1.600 rpm, velocidade máxima, em alta velocidade, $24 \mathrm{~km} / \mathrm{h}$, velocidade máxima, em baixa velocidade, $8,5 \mathrm{~km} / \mathrm{h}$, consumo médio de 23 litros de combustível por hora, capacidade de extração máxima $206 \mathrm{kN}(21.000 \mathrm{kp})$, eixo frontal rígido com cubo de marchas, rodado de pneus frontal 700/70x34, rodado de pneus traseiros 650/65x26.5. A garra de carregamento era da marca Super Grip, modelo SG420S, com área útil de 0,42 $\mathrm{m}^{2}$ e capacidade de carga de $5.500 \mathrm{~kg}$.

\section{Sistema de extração}

A derrubada e o processamento da madeira, etapa anterior à operação de extração com o forwarder, foi realizada por um harvester, que se deslocava em um eito de derrubada composto por três linhas de árvores. O sistema de colheita era de madeira curta, com toretes de 6 metros de comprimento. Os toretes eram dispostos perpendicularmente à linha de plantio, formando uma fila de feixes ao longo do eito de extração. 
A extração da madeira com o forwarder foi realizada numa distância média de 175 metros, sendo a distância mínima de 25 e a máxima de 350 metros, com o carregamento dos feixes de toretes dispostos nos dois lados do ramal, ou seja, por ambos os lados (direito e esquerdo) da máquina.

$\mathrm{O}$ deslocamento do forwarder sem carga era realizado em marcha à ré até o primeiro feixe a ser carregado no eito de extração. O carregamento dos toretes era efetuado, no interior do talhão, até completar a capacidade máxima do compartimento de carga. Em seguida o operador do forwarder iniciava o deslocamento com carga, em marcha avante, até o ponto de descarregamento situado às margens da estrada florestal. O descarregamento dos toretes iniciava-se com o acionamento da grua, retirando os toretes do compartimento de carga e colocando-os na pilha na margem da estrada florestal.

\section{Instrumental de medição}

A coleta de dados de tempos e movimentos foi efetuada pelo método de cronometragem de tempo contínuo. Esse método caracteriza-se pela medição do tempo sem detenção do cronômetro, isto é, de forma contínua. O ciclo operacional do forwarder foi subdividido pelas seguintes atividades parciais:

- carregamento: tempo despendido pela ação de carregamento dos toretes, incluso os ciclos da grua e acionamento da garra;

- descarregamento: tempo compreendido para a realização dos ciclos da grua e da garra para efetuar o descarregamento dos toretes;

- deslocamento com carga: deslocamento do forwarder em marcha à frente no ramal de extração, após a finalização do carregamento de toretes;

- deslocamento sem carga: tempo despendido para o deslocamento do forwarder em marcha à ré, com compartimento de carga vazio.

Foi utilizada a amostragem sistemática, sendo o número de ciclos operacionais estimados através da metodologia utilizada por Barnes (1968). Inicialmente realizou-se um estudo-piloto dos ciclos operacionais, buscando-se estabelecer o número mínimo de ciclos para um erro de amostragem admissível fixado em 5\%, a 95\% de probabilidade, através da equação 1.

$$
n \geq \frac{t^{2} \times C V^{2}}{E^{2}}
$$

Em que: $\mathrm{n}$ = número mínimo de ciclos operacionais necessários;

$\mathrm{t}=$ valor de $\mathrm{t}$, Student , no nível de probabilidade desejado e (n-1) graus de liberdade;

$\mathrm{CV}=$ coeficiente de variação $(\%)$;

$\mathrm{E}=$ erro admissível $(\%)$.

Após a estimativa dos tempos de deslocamento, os dados foram analisados em um programa estatístico, determinando-se as equações que melhor representam os dados ajustados às curvas-modelos que melhor explicam os procedimentos estudados.

\section{Eficiência operacional e disponibilidade mecânica}

A eficiência operacional é definida como o percentual do tempo das atividades efetivas, ou seja, engloba todas as atividades parciais que ocorreram repetitivamente durante o decurso do trabalho e que resultam em produção (Equação 2).

$$
E_{o}=\frac{H E \times 100}{H T}
$$

Em que: Eo = eficiência operacional (\%);

$\mathrm{HE}=$ tempo efetivo de trabalho (horas);

$\mathrm{HT}=$ tempo total de trabalho (horas).

A disponibilidade mecânica é definida como o percentual do tempo de trabalho delineado à máquina mecanicamente apta a desenvolver suas operações, o qual consiste em desconsiderar o tempo despendido para efetuar reparos ou manutenção (Equação 3). 


$$
D_{m}=100-\left(\frac{H M \times 100}{H T}\right)
$$

Em que: $\mathrm{Dm}=$ grau de disponibilidade mecânica $(\%)$;

$\mathrm{HM}=$ tempo de interrupção para efetuar reparos ou manutenção (horas);

$\mathrm{HT}=$ tempo total de trabalho (horas).

\section{Rendimento operacional da máquina}

Para realizar o cálculo do rendimento operacional da operação de extração de madeira com forwarder, foi utilizada a metodologia proposta por Simões (2008), conforme a equação 4:

$$
R=\frac{v}{T}
$$

Em que: $\mathrm{R}=$ rendimento operacional médio $\left(\mathrm{m}^{3} \cdot \mathrm{h}^{-1}\right)$;

$\mathrm{v}=$ volume, em metros cúbicos de madeira com casca extraída $\left(\mathrm{m}^{3}\right)$

$\mathrm{t}=$ tempo em horas $(\mathrm{h})$.

$\mathrm{O}$ volume de madeira foi obtido multiplicando-se o número de toretes pelo volume médio. O volume médio dos toretes foi determinado preliminarmente através de cubagem.

\section{Determinação dos custos operacionais}

Os custos operacionais do forwarder foram estimados em dólar por hora de trabalho (US\$.h ${ }^{-1}$ ), para o quais utilizou-se a taxa de câmbio de 1 dólar $=\mathrm{R} \$ 1,8032$, do dia 20/12/2007.

Para o cálculo dos custos fixos, foi considerado um valor de revenda de $52 \%$ do valor inicial da máquina florestal e vida útil de 4 anos. Os valores da máquina florestal e da mão de obra foram fornecidos pela empresa onde se desenvolveu o estudo. Foi utilizada a metodologia proposta pela American Society of Agricultural Engineers (ASAE, 2001).

\section{Custos fixos (CF)}

Os custos fixos foram aqueles que não variaram com a quantidade de horas da operação e independeram da produção da máquina florestal, sendo compostos por juros, custos de depreciação, abrigo, taxas e seguros.

a) Juros

Para a estimativa dos juros, foi utilizada uma taxa de $10 \%$ a.a., que se refere à utilizada pela empresa onde foi desenvolvido o estudo (Equação 5).

$$
J=\frac{V i+V f}{2 \times h} \times r
$$

Em que: $\mathrm{J}=$ custos com juros $\left(\mathrm{US} \$ . \mathrm{h}^{-1}\right)$;

$\mathrm{Vi}=$ valor inicial da máquina florestal (US\$);

$\mathrm{Vf}=$ valor final da máquina florestal (US\$);

$\mathrm{h}=$ horas efetivas de uso anual;

$\mathrm{r}=$ taxa de juros.

b) Depreciação

O custo de depreciação estimou a perda de valor no decorrer da vida útil da máquina florestal. A estimativa do custo de depreciação foi um procedimento utilizado para recuperar o investimento inicial da máquina, à medida que ela se torna obsoleta. Foi utilizado o método da depreciação linear, pois se considerou que os recursos financeiros correspondentes ficam no caixa da empresa com remuneração zero (Equação 6).

$$
D=\frac{V i-V f}{V u \times h}
$$


Em que: $\mathrm{D}=$ depreciação linear $\left(\mathrm{US} \$ \cdot \mathrm{h}^{-1}\right)$;

$\mathrm{Vi}=$ valor inicial da máquina florestal (US\$);

$\mathrm{Vf}=$ valor final de revenda ou de sucata da máquina florestal (US\$);

$\mathrm{Vu}=$ vida útil em anos;

$\mathrm{h}=$ horas de uso anual.

c) Abrigo

Foi calculado o custo de abrigo pago em função do local de armazenamento requerido pela máquina florestal. Foi considerado um fator de ajuste (FA) de $0,75 \%$ do valor inicial da máquina (Equação 7).

$$
C_{a}=\frac{V i \times F A}{h}
$$

Em que: $\mathrm{Ca}=$ custo de abrigo da máquina florestal $\left(\mathrm{US} \$ . \mathrm{h}^{-1}\right)$;

$\mathrm{Vi}=$ valor inicial da máquina florestal (US\$);

$\mathrm{FA}=$ fator de ajuste;

$\mathrm{h}=$ horas de uso por ano.

d) Taxas

Em função dos encargos administrativos, foi considerado um percentual do valor inicial da máquina, de acordo com o preconizado pela ASAE (2001). Dessa forma, o fator de ajuste (FA) foi de $1,0 \%$ (Equação 8).

$$
T=\frac{V i \times F A}{h}
$$

Em que: $\mathrm{T}=$ taxas de administração $\left(\mathrm{US} \$ \cdot \mathrm{h}^{-1}\right)$;

$\mathrm{Vi}=$ valor inicial da máquina florestal (US\$);

$\mathrm{FA}=$ fator de ajuste;

$\mathrm{h}=$ horas de uso por ano.

e) Seguros

$\mathrm{O}$ custo do seguro foi estimado para a proteção contra os riscos associados com roubo, incêndio e outros. Considerou-se um fator de ajuste (FA) de $0,25 \%$ do valor inicial da máquina (Equação 9).

$$
S=\frac{V i \times F A}{h}
$$

Em que: $\mathrm{Ca}=$ custo de seguro da máquina florestal $\left(\mathrm{US} \$ . \mathrm{h}^{-1}\right)$;

$\mathrm{Vi}=$ valor inicial da máquina florestal (US\$);

$\mathrm{FA}=$ fator de ajuste;

$\mathrm{h}=$ horas de uso por ano.

\section{Custos variáveis $(\mathrm{CV})$}

Os custos variáveis se modificaram proporcionalmente em relação ao nível das operações e do tempo despendido para a realização, ou seja, o custo operacional alterava-se de acordo com o desenvolvimento da operação. Foram considerados custos variáveis o custo do combustível, custos de mão de obra, lubrificação, reparos e manutenções.

a) Mão de obra

No custo horário de mão de obra estão inclusos os salários diretos e indiretos e todos os benefícios que os operadores recebem, com base na quantidade de horas trabalhadas, devido ao tempo despendido para abastecimento de combustível, lubrificação, manutenção, ausência da máquina no campo e transporte. Foi adotado um fator de ajuste de $25 \%$ proposto pela ASAE (2001), conforme a equação 10.

$$
C m o=\{W \times F A\}
$$


Em que: $\mathrm{Cmo}=$ custo de mão de obra $\left(\right.$ US $\left.\$ \cdot \mathrm{h}^{-1}\right)$;

$\mathrm{W}=$ salário médio $\left(\mathrm{US} \$ . \mathrm{h}^{-1}\right)$;

$\mathrm{FA}=$ fator de ajuste.

b) Combustíveis

O custo de combustível foi estimado com base na quantidade de óleo diesel consumido pela máquina florestal (Equação 11).

A máquina florestal empregada no estudo possui um módulo de controle eletrônico do motor, o que possibilita armazenar e transmitir informações sobre o consumo médio de combustível, sendo essas informações fornecidas preliminarmente pela empresa. Esse consumo foi em função da potência do motor, tipo de combustível, capacidade operacional e operação desenvolvida, entre outros fatores.

$$
C c=Q m \times P c
$$

Em que: $\mathrm{Cc}=$ custo de combustível $\left(\mathrm{US} \$ . \mathrm{h}^{-1}\right)$;

$\mathrm{Qm}=$ consumo médio de diesel $\left(1 . \mathrm{h}^{-1}\right)$;

$\mathrm{Pc}=$ Preço do óleo diesel $\left(\mathrm{US} \$ \cdot 1^{-1}\right)$.

c) Lubrificação

Devido à demanda de grande quantidade de trabalho, não justificada em função dos pequenos valores envolvidos para se determinar a quantidade dos produtos consumidos e a cada quanto tempo se faz a lubrificação da máquina, a empresa não realiza o controle dessas informações.

Com base em indicadores médios de consumo e das informações técnicas do fabricante, os custos de lubrificantes foram estimados com fator de ajuste de 15\% dos custos do combustível consumido por hora de trabalho, conforme a metodologia proposta pela ASAE (2001).

Nos custos de lubrificação estão inclusos os dispêndios com óleo de motor, óleo de transmissão, graxas e filtros, estimados através da equação 12 .

$$
C l=F A \times C c
$$

Em que: $\mathrm{Cl}=$ custo de lubrificação $\left(\mathrm{US} \$ \cdot \mathrm{h}^{-1}\right)$;

$\mathrm{FA}=$ fator de ajuste;

$\mathrm{Cc}=$ preço do óleo diesel $\left(\mathrm{US} \$ \cdot \mathrm{l}^{-1}\right)$.

d) Reparos e manutenção

Os custos de reparo e manutenção incidem devido ao uso em condições normais ou em consequência de desgastes dos componentes, acidentes ou deterioração natural da máquina florestal, representados na equação 13 .

$$
c_{r m}=\frac{\left(P \times F R_{1}\left(\frac{h+u}{1000}\right)^{F R_{2}}\right)-\left(P \times F R_{1}\left(\frac{h}{1000}\right)^{F R_{2}}\right)}{u}
$$

Em que: $\mathrm{Crm}=$ custos de reparo e manutenção $\left(\right.$ US $\left.\$ . \mathrm{h}^{-1}\right)$;

$\mathrm{P}=$ valor inicial da máquina florestal (US\$);

FR1 = fator de reparo $1(0,003)$;

$\mathrm{h}=$ horas de uso acumulada, até o início do ano em análise;

$\mathrm{FR} 2$ = fator de reparo $2(2,0)$;

$\mathrm{u}=$ horas de uso no ano em análise.

\section{Custo operacional}

Esse custo foi obtido pelo somatório dos custos fixos e variáveis relativos à máquina avaliada, de acordo com o descrito na equação 14 .

$$
\mathrm{Cot}=\mathrm{CF}+\mathrm{CV}
$$

Em que: $\mathrm{Cot}=$ custo operacional total da máquina florestal $\left(\mathrm{US} \$ . \mathrm{h}^{-1}\right)$;

$\mathrm{CF}=$ custos fixos $\left(\mathrm{US} \$ . \mathrm{h}^{-1}\right)$;

$\mathrm{CV}=$ custos variáveis $\left(\mathrm{US} \$ \cdot \mathrm{h}^{-1}\right)$. 


\section{Custos de extração da madeira}

O custo de extração da madeira (US\$. $\mathrm{m}^{-3}$ ) foi obtido através da divisão dos custos operacionais pelo rendimento operacional efetivo da máquina florestal, conforme a equação 15, utilizada por Simões (2008).

$$
C c m=\frac{\operatorname{CopMF}}{R}
$$

Em que: $\mathrm{Ccm}=$ custo de extração da madeira $\left(\mathrm{US} \$ . \mathrm{m}^{-3}\right)$;

CopMF = custo operacional da máquina florestal $\left(\mathrm{US} \$ . \mathrm{h}^{-1}\right)$;

$\mathrm{R}=$ rendimento operacional $\left(\mathrm{m}^{3} \cdot \mathrm{h}^{-1}\right)$.

\section{RESULTADOS E DISCUSSÃO}

Os resultados apresentados referem-se somente às atividades parciais efetivas que compõem o ciclo de trabalho do forwarder.

Durante o estudo, foram processados 11.338 toretes e um volume total de $931,98 \mathrm{~m}^{3}$. O comprimento médio dos toretes era de 6 metros e o volume médio $0,0822 \mathrm{~m}^{3}$. Foram estudados 39 ciclos de trabalho, número superior ao calculado como necessário para um erro de amostragem admissível fixado em $5 \%$ a $95 \%$ de probabilidade.

\section{Estudo de tempos e movimentos}

$\mathrm{Na}$ figura 1 são apresentados os percentuais dos tempos das atividades parciais do forwarder. Entre as atividades parciais efetivas que compuseram o ciclo operacional, verificou-se que a atividade parcial que consumiu maior parte do tempo do ciclo operacional era o carregamento, com $50,57 \%$ do tempo total. A segunda atividade parcial foi o descarregamento, com $27,83 \%$, seguida da atividade parcial deslocamento com carga, com $13,35 \%$, e pelo deslocamento sem carga, com $8,25 \%$.

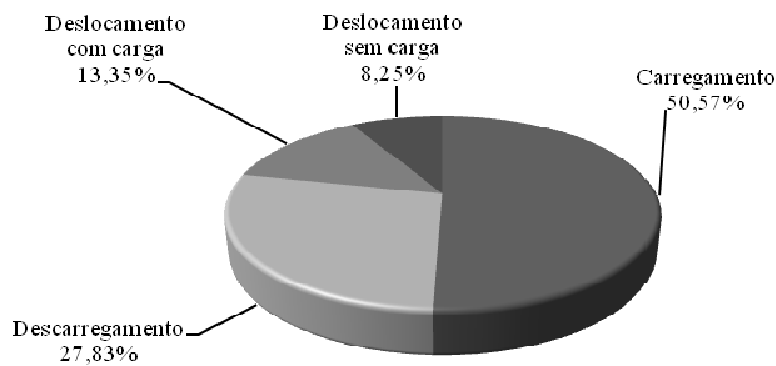

Figura 1. Percentual de tempos das atividades parciais do forwarder.

Figure 1. Percent of times the partial activities of forwarder.

O percentual do tempo despendido para carregar foi superior ao citado por Bantel (2006), que em seu estudo avaliando a extração de toretes de $5,5 \mathrm{~m}$ de comprimento de eucalipto com forwarder em floresta de primeira e segunda rotação, para distâncias médias de extração de 150 metros, determinou percentuais de 43,02 e 46,91, respectivamente. Esse fato pode ser explicado em função do comprimento dos toretes. Portanto, o comprimento dos toretes é uma variável que pode influenciar no tempo despendido para se efetuar o carregamento.

Minette et al. (2004), que trabalharam com a extração de madeira de $6 \mathrm{~m}$ de comprimento, para distâncias médias de 122 e 129 metros, definiram para o tempo de descarregar percentuais de 28,17 e 28,33 , valores próximos ao deste estudo. Na tabela 1 são apresentadas as equações que relacionam os tempos de deslocamento com carga e sem carga com a distância. Os sinais positivos do coeficiente da variável distância nos dois modelos indicam que o aumento na distância média ocasionou aumento nos tempos de ambos os deslocamentos. 


\section{Eficiência operacional e disponibilidade mecânica}

Ponderando todas as atividades parciais efetivas, foi observado para o forwarder uma eficiência operacional de $82,21 \%$ e disponibilidade mecânica de $88,35 \%$.

Tabela 1. Equações estimadas para os tempos dos deslocamentos com carga e sem carga do forwarder. Table 1. Equations estimated for the with load and unloaded displacement times of the forwarder.

\begin{tabular}{lcc}
\hline Equação estimada & $\mathbf{R}^{\mathbf{2}}$ & C.V. $(\%)$ \\
\hline DCC $=-0,0989^{*}+2,5410^{*}$ x Dist. & 0,99 & 0,28 \\
DSC $=0,0319 *+2,3098^{*}$ x Dist. & 0,99 & 0,29 \\
\hline DCC: deslocamento com carga (seg.); DSC: deslocamento sem carga (seg.); R $\mathrm{R}^{2:}$ coeficiente de \\
determinação; Dist.: distância (m); CV: coeficiente de variação; *: significativo a 5\%.
\end{tabular}

\section{Rendimento operacional}

O rendimento operacional efetivo do forwarder foi de $94,89 \mathrm{~m}^{3} \cdot \mathrm{h}^{-1}$, para uma distância média de extração de 175 metros. Esse valor foi superior ao citado por Bantel (2006), que determinou uma produtividade de 77,35 e $84,26 \mathrm{~m}^{3} \cdot \mathrm{h}^{-1}$ em sistemas de extração com distância média de $150 \mathrm{~m}$, toras de $5,5 \mathrm{~m}$ e demais fatores semelhantes ao deste estudo. Esse fato corrobora que a operação de extração com toras mais longas é a mais produtiva, o que também foi constatado por Santos; Machado (2001). Entretanto pode haver um decréscimo na produtividade em função do aumento da distância da extração.

\section{Custo operacional}

Diante do somatório dos custos fixos e variáveis, o forwarder apresentou um custo operacional de US\$ 92.47 por hora de trabalho.

A figura 2 apresenta a distribuição percentual dos custos operacionais obtidos para o forwarder. O custo fixo correspondeu a $35,83 \%$, e o custo variável representou $64,17 \%$.

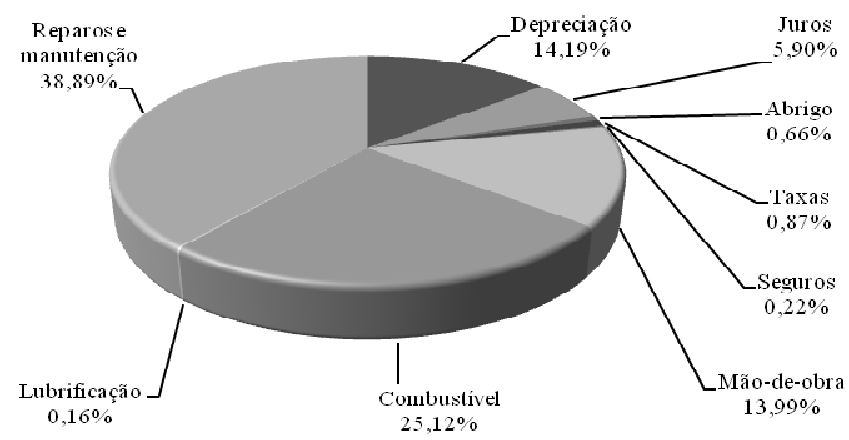

Figura 2. Distribuição percentual do custo operacional do forwarder.

Figure 2. Percent distribution of the forwarder operational cost.

O forwarder empregado no estudo apresentava mais de 14.000 horas de uso, fato que pode explicar porque o custo de reparo e manutenção foi o item que mais impactou o custo operacional. Esse comportamento permite concluir que, a partir de uma determinada quantidade de horas de uso, os custos com reparos e manutenção serão superiores aos demais itens que compõem os custos operacionais.

\section{Custo de extração}

Considerado o rendimento operacional efetivo, o custo de extração resultou em US\$ 0.97 por metro cúbico de madeira com casca, numa distância média de 175 metros.

Na Figura 3 é apresentada uma curva do tipo exponencial que relaciona o custo de extração em função do rendimento operacional efetivo do forwarder. O custo de extração variou de US\$9.25 a US\$ 0.92 para uma faixa de rendimento operacional de $10 \mathrm{~m}^{3} \cdot \mathrm{h}^{-1}$ a $100 \mathrm{~m}^{3} \cdot \mathrm{h}^{-1}$, com melhores condições de operação. Foi traçada uma linha de tendência que gerou a referida equação. 


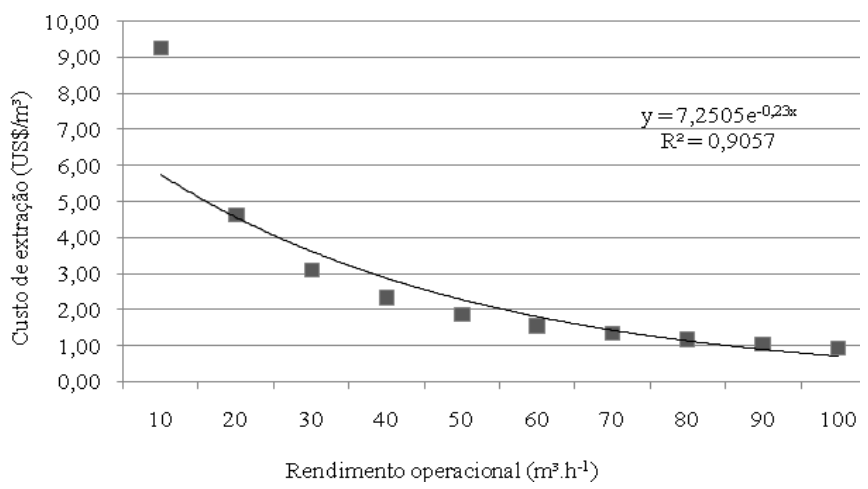

Figura 3. Custo de extração em função do rendimento operacional.

Figure 3. Extraction cost in function of the operational performance.

\section{CONCLUSÕES}

- As atividades parciais de carregamento e de descarregamento contíguas representaram aproximadamente $80 \%$ do tempo total do ciclo operacional do forwarder.

- O custo de extração de madeira foi inversamente proporcional ao rendimento operacional.

- O custo de reparo e manutenção foi o item de maior ônus entre os demais que compuseram o custo operacional.

\section{REFERÊNCIAS}

AMERICAN SOCIETY OF AGRICULTURAL ENGINEERS. ASAE standards 2001: machinery, equipment and buildings: operating costs. Ames, Iowa, USA, 2001. p. 164-226. (ASAE D472-3).

BANTEL, C. A. Análise de extração de madeira de eucalipto com forwarder em floresta de primeira e segunda rotação. Botucatu, 2006. 126 f. Dissertação (mestrado). Faculdade de Ciências Agronômicas, Universidade Estadual Paulista.

BARNES, R. M. Motion and time study: design and measurement of work. 6. ed. New York: John Willey and Sons, $1968.799 \mathrm{p}$.

CUNHA, A. R.; KLOSOWSKI, E. S.; GALVANI, E.; ESCOBEDO, J. F.; MARTINS, D. Classificação climática para o município de Botucatu, SP, segundo Köppen. In: SIMPÓSIO EM ENERGIA NA AGRICULTURA, 1., 1999, Botucatu. Anais... Botucatu: SINERGIA, 1999. v. 2, p. 487-489.

MALINOVSKI, R. A.; MALINOVSKI, R. A.; MALINOVSKI, J. R.; YAMAJI, F. M. Análise das variáveis de influência na produtividade das máquinas de colheita de madeira em função das características físicas do terreno, do povoamento e do planejamento operacional florestal. Revista Floresta, Curitiba, v. 36, n. 2, p. 169-182, mai./ago, 2006.

MINETTE, L. J.; MOREIRA, F. M. T.; SOUZA, A. P.; MACHADO, C. C.; SILVA, K. R. Análise técnica e econômica do forwarder em três subsistemas de colheita de florestas de eucalipto. Revista Árvore, Viçosa, MG, v. 28, n. 1, p. 91-97, 2004.

MINETTE, L. J. Avaliação técnica e econômica dos tratores florestais transportadores (forwarders), na extração de madeira de eucalipto. Viçosa: UFV, 1988. 77 p. Tese (Mestrado em Ciência Florestal) Universidade Federal de Viçosa, 1988.

MINETTE, L. J.; SILVA, E. N.; MIRANDA, G. M.; SOUZA, A. P.; FIEDLER, N. C. Avaliação técnica da operação de extração de Eucalyptus spp. utilizando o trator autocarregável e o trator florestal 
transportador "forwarder" na região sul da Bahia. Engenharia na Agricultura, Viçosa, MG, v. 16, n. 3, p. 312-317, 2008.

SANTOS, S. L. M.; MACHADO, C. C. Avaliação técnica e econômica da extração de madeira com forwarder em diferentes volumes por árvore e comprimentos de toras. Revista Madera y Bosques, Cidade do México, v. 7, n. 2, p. 87-94, 2001.

SEIXAS, F. Planejamento e estudo de sistema de exploração florestal. IPEF, Piracicaba, v. 34, p. 25-30, 1986.

SIMÕES, D. Avaliação econômica de dois sistemas de colheita florestal mecanizada de eucalipto. 2008. 105 f. Dissertação (Mestrado em Agronomia/Energia na Agricultura) - Faculdade de Ciências Agronômicas/Universidade Estadual Paulista, Botucatu, SP, 2008. 